\title{
Visual decompositions of Coxeter groups
}

\author{
Michael Mihalik and Steven Tschantz
}

\begin{abstract}
A Coxeter system is an ordered pair $(W, S)$ where $S$ is the generating set in a particular type of presentation for the Coxeter group $W$. A subgroup of $W$ is called special if it is generated by a subset of $S$. Amalgamated product decompositions of a Coxeter group having special factors and special amalgamated subgroup are easily recognized from the presentation of the Coxeter group. If a Coxeter group is a subgroup of the fundamental group of a given graph of groups, then the Coxeter group is also the fundamental group of a graph of special subgroups, where each vertex and edge group is a subgroup of a conjugate of a vertex or edge group of the given graph of groups. A vertex group of an arbitrary graph of groups decomposition of a Coxeter group is shown to split into parts conjugate to special groups and parts that are subgroups of edge groups of the given decomposition. Several applications of the main theorem are produced, including the classification of maximal FA subgroups of a finitely generated Coxeter group as all conjugates of certain special subgroups.
\end{abstract}

Mathematics Subject Classification (2000). 20F55, 20E08, $20 \mathrm{E} 28$.

Keywords. Coxeter group, group actions on trees, graph of groups decompositions, FA groups, accessibility of groups.

\section{Introduction}

We take a Coxeter presentation to be given as

$$
P=\left\langle S:(s t)^{m(s, t)}(s, t \in S, m(s, t)<\infty)\right\rangle,
$$

where $m: S^{2} \rightarrow\{1,2, \ldots, \infty\}$ is such that $m(s, t)=1$ iff $s=t$ and $m(s, t)=$ $m(t, s)$. In the group with this presentation, the elements of $S$ represent distinct elements of order 2 and a product $s t$ of generators has order $m(s, t)$. A Coxeter group $W$ is a group having a Coxeter presentation and a Coxeter system $(W, S)$ is a Coxeter group $W$ with generating subset $S$ corresponding to the generators in a Coxeter presentation of $W$. When the order of the product of a pair of generators is infinite there will be no defining relator for that pair of generators and we will say that the generators are unrelated. A Coxeter group $W$ belongs to some Coxeter system 
$(W, S)$, and though $S$ need not be uniquely determined up to an automorphism of $W$, we often take such an $S$ as given. Our basic reference for Coxeter groups is Bourbaki [3]. A special subgroup of a Coxeter system $(W, S)$, is a subgroup of $W$ generated by a subset of $S$ (see [5]). If $W^{\prime}$ is the special subgroup generated by $S^{\prime} \subseteq S$ in a Coxeter system $(W, S)$, then $\left(W^{\prime}, S^{\prime}\right)$ is also a Coxeter system.

The information given by a Coxeter presentation may be conveniently expressed in the form of a labeled graph. We define the presentation diagram of the system $(W, S)$ to be the labeled graph $\Gamma(W, S)$ with vertex set $S$, and an (undirected) edge labeled $m(s, t)$ between distinct vertices $s$ and $t$ when $m(s, t)<\infty$. The connected components of the presentation diagram $\Gamma(W, S)$ correspond to special subgroups which are the factors in a free product decomposition of $W$. (This is in contrast to a Coxeter graph with vertex set $S$ and labeled edges when $m(s, t) \neq 2$, and having components corresponding to direct product factors of $W$. The Coxeter graph is not used in this paper.) The presentation diagram of the special subgroup of $W$ generated by a subset $S^{\prime} \subseteq S$ is the induced subgraph of $\Gamma(W, S)$ with vertex set $S^{\prime}$ (and in this sense, special subgroups could as well be termed visual subgroups since we can see the presentation diagram of such a subgroup in $\Gamma(W, S))$.

Suppose that $\Gamma(W, S)=\Gamma_{1} \cup \Gamma_{2}$ is a union of induced subgraphs and let $\Gamma_{0}=\Gamma_{1} \cap \Gamma_{2}$ (so vertices and edges of $\Gamma(W, S)$ are in $\Gamma_{1}$ or $\Gamma_{2}$ or both, and $\Gamma_{0}$ is the induced subgraph consisting of the vertices and edges in both). Equivalently, suppose $\Gamma_{0}$ is an induced subgraph with $\Gamma(W, S)-\Gamma_{0}$ having at least two components, $\Gamma_{1}$ is $\Gamma_{0}$ together with some of these components and $\Gamma_{2}$ is $\Gamma_{0}$ together with the other components. We say in this case that $\Gamma_{0}$ separates $\Gamma(W, S)$ (separates it into at least two components). Then it is evident from the Coxeter presentation that $W$ is an amalgamated product of special subgroups corresponding to $\Gamma_{1}$ and $\Gamma_{2}$ over the special subgroup corresponding to $\Gamma_{0}$. Amalgamated product decompositions with special factors and special amalgamated subgroup are easily seen in the presentation diagram and we call such an amalgamated product a visual splitting of $W$. Other amalgamated product decompositions may also be possible, and we want to understand such splittings in terms of visual splittings.

More generally, we are interested in when a Coxeter group $W$ can be realized as the fundamental group of a graph of groups (as explained in the next section). We show that the graph must actually be a tree and so this generalizes amalgamated products. We say that $\Psi$ is a visual graph of groups decomposition of $W$ (for a given $S$ ), if each vertex and edge group of $\Psi$ is a special subgroup of $W$, the injections of each edge group into its endpoint vertex groups are given simply by inclusion, and the fundamental group of $\Psi$ is isomorphic to $W$ by the homomorphism induced by the inclusion map of vertex groups into $W$. A sequence of compatible visual splittings of $W$ will result in such a decomposition. Our main result shows that an arbitrary graph of group decomposition of a Coxeter group can be refined (in a certain sense) to a visual graph of groups decomposition. 
Theorem 1 (Main theorem). Suppose that $(W, S)$ is a Coxeter system and $W$ is a subgroup of the fundamental group of a graph of groups $\Lambda$. Then $W$ has a visual graph of groups decomposition $\Psi$ where each vertex group of $\Psi$ is a subgroup of a conjugate of a vertex group of $\Lambda$, and each edge group of $\Psi$ is a subgroup of a conjugate of an edge group of $\Lambda$. Moreover, $\Psi$ can be taken so that each special subgroup of $W$ that is a subgroup of a conjugate of a vertex group of $\Lambda$ is a subgroup of a vertex group of $\Psi$.

For $(W, S)$ and $\Lambda$ as in Theorem 1 and $\Psi$ satisfying the full conclusion of Theorem 1 (including the moreover clause), we say that $\Psi$ is a $(W, S)$-visual decomposition from $\Lambda$. Suppose that $G$ is a group decomposed as $A *_{C} B$. If $H$ is a subgroup of $B$, then $\langle A \cup H\rangle$ decomposes as $A *_{C}\langle C \cup H\rangle$ (consider the action of $\langle A \cup H\rangle$ on the Bass-Serre tree for $\left.A *_{C} B\right)$. Furthermore, the decomposition $\left(A *_{C}\langle C \cup H\rangle\right)\langle C \cup H\rangle B$ reduces to $A *_{C} B$, so $G$ decomposes as $\langle A \cup H\rangle *_{\langle C \cup H\rangle} B$. In this way some visual decompositions of Coxeter groups can be "artificially" altered to decompositions that have no visual vertex or edge groups. In Section 3, we exhibit a Coxeter system $(W, S)$ and a reduced graph of groups decomposition $\Lambda_{N}$, for $(W, S)$ with $N$ vertices for any positive integer $N$. Even so, the following theorem defines limits on how far an arbitrary graph of groups decomposition for a finitely generated Coxeter system can stray from a visual decomposition for that system.

Theorem 2. Suppose that $(W, S)$ is a finitely generated Coxeter system, $\Lambda$ is a graph of groups decomposition of $W$ and $\Psi$ is a reduced graph of groups decomposition of $W$ such that each vertex group of $\Psi$ is a subgroup of a conjugate of a vertex group of $\Lambda$. Then for each vertex $V$ of $\Lambda$, the vertex group $\Lambda(V)$ has a graph of groups decomposition $\Phi_{V}$ such that each vertex group of $\Phi_{V}$ is either

(1) conjugate to a vertex group of $\Psi$, or

(2) a subgroup of $v \Lambda(E) v^{-1}$ for some $v \in \Lambda(V)$ and $E$ some edge of $\Lambda$ adjacent to $V$.

When $\Psi$ is visual, vertex groups of the first type in Theorem 2 are visual. Those of the second type seem somewhat artificial.

It is easy to recognize whether a finitely generated Coxeter group is 2-ended or infinite ended, by refining Stallings' theorem (from [18]) to a visual splitting theorem. A Dunwoody decomposition of a finitely generated Coxeter group is a graph of groups with finite or 1-ended vertex groups and finite edge groups. These refine to a visual Dunwoody decomposition, and we get a simple argument for why finitely generated Coxeter groups are accessible with respect to splittings over finite groups (as in [10]). In separate papers [14] and [15], we developed the fundamental ideas of this paper to prove a strong accessibility result for Coxeter groups with respect to splittings over "minimal" splitting subgroups, and a JSJ result for splittings of Coxeter groups over 
virtually abelian groups. At the bottom level, the strong accessibility result is a visual version of Dunwoody's result in the Coxeter setting.

J.-P. Serre gives an account of FA groups in [17]. In particular, an FA group has no nontrivial splittings. We apply the main theorem to show that the maximal FA subgroups of a finitely generated Coxeter group $W$ are those conjugate to a special subgroup with presentation diagram a maximal complete subgraph of $\Gamma(W, S)$.

Our results identify certain properties of a Coxeter group recognizable directly from a particular presentation of the group, properties apparent in the presentation diagram. There can be different Coxeter systems $(W, S)$ and $\left(W, S^{\prime}\right)$ where $S^{\prime}$ is not simply a conjugate of $S$ (and may not even correspond under any automorphism of $W$ ), and so conjugates of special subgroups with respect to $S$ need not correspond to conjugates of special subgroups with respect to $S^{\prime}$. However, these results do imply that certain special subgroups are, up to conjugation, special for any Coxeter system. This investigation thus has significant application to rigidity questions and the determination of when Coxeter groups are isomorphic.

As a final applications of these ideas we give a visual classification of finitely generated virtually free Coxeter groups in Section 8.

\section{Graphs of groups}

Our main tool in this investigation is the connection between group actions on trees and fundamental groups of graphs of groups. As reference the reader is referred to [9] and [17]. We review some of the pertinent definitions and results.

A graph of groups $\Lambda$ consists of a set $V(\Lambda)$ of vertices, a set $E(\Lambda)$ of edges, and maps $\iota, \tau: E(\Lambda) \rightarrow V(\Lambda)$ giving the initial and terminal vertices of each edge in a connected graph, together with vertex groups $\Lambda(V)$ for $V \in V(\Lambda)$, edge groups $\Lambda(E)$ for $E \in E(\Lambda)$, with $\Lambda(E) \subset \Lambda(\iota(E))$ and an injective group homomorphism $t_{E}: \Lambda(E) \rightarrow \Lambda(\tau(E))$, called the edge map of $E$ and denoted by $t_{E}: g \mapsto g^{t_{E}}$. The fundamental group $\pi_{1}(\Lambda)$ of a graph of groups $\Lambda$ is the group with presentation having generators the disjoint union of $\Lambda(V)$ for $V \in V(\Lambda)$, together with a symbol $t_{E}$ for each edge $E \in E(\Lambda)$, and having as defining relations the relations for each $\Lambda(V)$, the relations $g t_{E}=t_{E} g^{t_{E}}$ for $E \in E(\Lambda)$ and $g \in \Lambda(\iota(E))$, and relations $t_{E}=1$ for $E$ in a given spanning tree of $\Lambda$ (the result, up to isomorphism, is independent of the spanning tree taken).

An amalgamated product $A{ }^{*} C B$ is realized as the fundamental group of the graph of groups with 2 vertices having vertex groups $A$ and $B$, a single edge between, with edge group (the image in $A$ of) $C$, and edge map $t_{E}$ determined by the injection of the edge group into $B$. Similarly, an HNN-extension of $A$ by $t_{E}: C \rightarrow A$ is realized as the fundamental group of a graph of groups with a single vertex and single edge. In general, the fundamental group of a graph of groups can be understood as taking amalgamated products of the vertex groups along the edge groups in the spanning tree, 
followed by HNN-extensions over the remaining edge groups. The $t_{E}$ for edges not in the spanning tree correspond to a stable letter of an HNN extension. For edges in the spanning tree the relations amount to identifying $\Lambda(E)$ in $\Lambda(\iota(E))$ with its image $\Lambda(E)^{t_{E}}$ in $\Lambda(\tau(E))$ as in an amalgamated product. Each vertex and edge group of a graph of groups $\Lambda$ injects into the fundamental group of $\Lambda$ (Britton's lemma) and we usually identify these with the corresponding subgroups of the fundamental group of $\Lambda$.

A graph of groups on a graph which is not simply connected will have $\mathbb{Z}$ as a homomorphic image (take a $t_{E}$ not in the spanning tree to 1 and all other generators to 0 ). Since the generators of a Coxeter group are of order 2 , a homomorphism into $\mathbb{Z}$ must have trivial image. Thus if a Coxeter group is the fundamental group of a graph of groups the graph must be a tree and the group arises as successive amalgamated products. In working with such trees of groups we will often simply assume suitable identifications have been made and the edge maps are simply inclusion maps.

Given a graph of groups $\Lambda$, the Bass-Serre tree for $\Lambda$ is defined with vertices the disjoint union over $V \in V(\Lambda)$ of the different cosets $g \Lambda(V)$ of $\Lambda(V)$ in $\pi_{1}(\Lambda)$, and edges the disjoint union over $E \in E(\Lambda)$ of the different cosets $g \Lambda(E)$ of $\Lambda(E)$ in $\pi_{1}(\Lambda)$, taken with $\iota(g \Lambda(E))=g \Lambda(\iota(E))$ and $\tau(g \Lambda(E))=g \Lambda(\tau(E))$. The BassSerre tree is in fact a tree and the fundamental group of $\Lambda$ acts on this tree by taking for $h \in \pi_{1}(\Lambda), h(g \Lambda(V))=(h g) \Lambda(V)$ and $h(g \Lambda(E))=(h g) \Lambda(E)$.

If a group $G$ acts on a tree $T$ (as a directed graph, the action preserving the orientation of edges), then a transversal for this action consists of a vertex and edge from each orbit of the action of $G$ on vertices and edges. There must always exist a transversal having a spanning subtree such that each other edge of the transversal originates in the subtree. (For instance, if $\Lambda$ has a single vertex and single edge, giving $G=\pi_{1}(\Lambda)$ as an HNN extension, then $G$ acts on the Bass-Serre tree $T$ for $\Lambda$ with transversal consisting of a single vertex and an edge originating at that vertex, that does not include the terminal vertex and has spanning subtree just the single vertex.) From such a transversal, a graph of groups is defined by taking as graph the quotient of $T$ under the action of $G$, and taking vertex and edge groups to be the stabilizers of the corresponding vertices and edges in the transversal. For edges in the spanning subtree of the transversal the edge maps are given by inclusion. An edge of the transversal not in the spanning subtree connects a vertex in the transversal to a translate by an element $g \in G$ of a vertex in the transversal and the edge map is given by conjugation by $g$. Then the fundamental group of this graph of groups is naturally isomorphic to $G$ by the homomorphism extending the inclusion map of vertex groups into $G$. Choosing a different transversal in $T$ gives rise to a different graph of groups decomposition of $G$ with vertex groups each conjugate to vertex groups of the first graph of groups, i.e., having isomorphic vertex groups but corresponding with different subgroups of $G$.

Suppose that $\Lambda$ is a graph of groups and $X$ is a subset of the edges in $\Lambda$. Contracting the graph of $\Lambda$ along the edges in $X$ gives rise to a graph whose vertices are the equivalence classes of vertices modulo the equivalence relation defined by identifying 
endpoints of edges in $X$, taking edges $E \in E(\Lambda)-X$ with endpoints the equivalence classes of endpoints of $E$. Define a graph of groups $\Lambda^{\prime}$ on this graph, having the same fundamental group as $\Lambda$, by taking as vertex groups $\Lambda^{\prime}(V)$ the fundamental group of the graph of groups with vertices of $\Lambda$ identified to $V$ and edges in $X$ between these vertices, with edge groups $\Lambda^{\prime}(E)$ corresponding to the remaining edge groups in $\Lambda$ with corresponding edge maps. We call $\Lambda^{\prime}$ the graph of groups resulting from collapsing the edges $X$ in $\Lambda$. If $\Lambda$ is a tree of groups, then collapsing edges in $\Lambda$ results in another tree of groups.

A graph of groups will be called reduced if no edge group is equal to its originating vertex group nor has image equal to its terminating vertex group, except for an edge which is a loop at a vertex. If a graph of groups is not reduced, then we may collapse a vertex across an edge, where the edge group is (or has image) the same as the endpoint vertex group, giving a smaller graph of groups with vertex and edge groups among the original vertex and edge groups and having the same fundamental group. Repeated reductions of this sort in a finite graph of groups must eventually end with a reduced graph of groups all of whose vertex and edge groups were present in the original graph of groups and having the same fundamental group.

The following is well known. We include a proof as this result is frequently referenced throughout the article.

Lemma 3. Suppose that $\Lambda$ is a reduced graph of groups decomposition of a group $G$, the underlying graph for $\Lambda$ is a tree, $V$ and $U$ are vertices of $\Lambda$, and $g \Lambda(V) g^{-1} \subset$ $\Lambda(U)$ for some $g \in G$, then $V=U$ and $g \in \Lambda(V)$.

Proof. If $U \neq V$ or if $U=V$ and $g \notin \Lambda(V)$, then in the Bass-Serre tree for $\Lambda, \Lambda(V)$ stabilizes the distinct vertices $\Lambda(V)$ and $g^{-1} \Lambda(U)$. But then $\Lambda(V)$ also stabilizes a geodesic path between these vertices and hence stabilizes the first edge $h \Lambda(E)$ for an edge $E$ of $\Lambda$ at $V$. This would mean that $\Lambda(V)$ is equal to $\Lambda(E)$ (or its image in $\Lambda(V)$ ), and we could collapse $V$ across $E$, contradicting that $\Lambda$ was reduced.

In the other direction, given a graph of groups $\Lambda$ and a graph of groups decomposition of a vertex group $\Lambda(V)$ as $\pi_{1}(\Phi)$, we would like to see when $\Lambda$ results from collapsing out $\Phi$ in a larger graph of groups $\Lambda^{\prime}$. Say that $\Phi$ is a compatible decomposition of $\Lambda(V)$ if each edge group of $\Lambda$ incident at $V$ is a subgroup of a $\Lambda(V)$ conjugate of a vertex group of $\Phi$. In general then, given $\Lambda$ and $\Phi$ a compatible decomposition of $\Lambda(V)$, construct $\Lambda^{\prime}$ by replacing $V$ in $\Lambda$ by $\Phi$ and attaching edges of $\Lambda$ incident at $V$ instead to vertices in $\Phi$ with edge groups and edge maps appropriately adjusted. So each such edge group has image in a vertex group of $\Phi$ (for edges ending in $\Phi$ in $\Lambda^{\prime}$ or $V$ in $\Lambda$ ) or is an isomorphic subgroup of a vertex group of $\Phi$ (for edges originating in $\Phi$ in $\Lambda^{\prime}$ or $V$ in $\left.\Lambda\right)$. This adjustment of edge maps means that the image in $\pi_{1}\left(\Lambda^{\prime}\right)$ of a vertex group of $\Lambda^{\prime}$ not in $\Phi$ is conjugate to the image of the corresponding vertex group of $\Lambda$ in $\pi_{1}(\Lambda)=\pi_{1}\left(\Lambda^{\prime}\right)$. 
Alternatively, the Bass-Serre tree $T^{\prime}$ for $\Lambda^{\prime}$ can be constructed from the BassSerre tree for $\Lambda$ with each coset of $\Lambda(V)$ replaced by the Bass-Serre tree for $\Phi$ with a $\pi_{1}(\Lambda)$ group action. Contracting the edges in the orbit of $\Phi$ in $T^{\prime}$ gives $T$, and a transversal of $T^{\prime}$ with a spanning subtree contracts to a transversal of $T$ with a spanning subtree. Starting with a transversal with spanning subtree of $T$ and a transversal with spanning subtree of the Bass-Serre tree for $\Phi$ we may need to translate the parts of the $T$ transversal in different components of $T-\{V\}$ so that they attach to the transversal of the Bass-Serre tree for $\Phi$ in $T^{\prime}$. The result is still a transversal of $T$ with the same quotient graph as $\Lambda$ but with vertex groups identified with conjugates of the vertex groups of $\pi_{1}(\Lambda)$.

Suppose that $(W, S)$ is a Coxeter system. A visual graph of groups decomposition $\Psi$ of $W$ has special vertex and edge groups, edge maps given by inclusion, and is such that the inclusion of vertex groups in $W$ extends to an isomorphism of $\pi_{1}(\Psi)$ with $W$. As noted above, $\Psi$ is a tree. In terms of presentation diagrams, $\Gamma(W, S)$ must be the union of the subdiagrams corresponding to the vertex groups of $\Psi$, with the edge groups corresponding to the intersections of adjacent vertex subdiagrams. To understand in visual terms exactly when a graph of special subgroups has fundamental group isomorphic to $W$ we have the following essential lemma.

Lemma 4. Suppose that $(W, S)$ is a Coxeter system. A graph of groups $\Psi$ with graph a tree, where each vertex group and edge group is a special subgroup and each edge map is given by inclusion, is a visual graph of groups decomposition of $W$ iff each edge in the presentation diagram of $W$ is an edge in the presentation diagram of a vertex group and, for each generator $s \in S$, the set of vertices and edges with groups containing $s$ is a nonempty subtree in $\Psi$.

Proof. Suppose that $\Psi$ is a visual graph of groups decomposition of $W$ so that the homomorphism $\psi$ extending the inclusion map on vertex groups is an isomorphism of $\pi_{1}(\Psi)$ and $W$. Since each vertex group is generated by the elements of $S$ it contains, the image of $\psi$ is generated by the set of all $s \in S$ that belong to some vertex group. Since a proper subset of $S$ generates a proper subgroup of $W$, each element of $S$ must be an element of a vertex group of $\Psi$. Let $T$ be the Bass-Serre tree for $\Psi$. Then since the edge maps are inclusions, the subgraph of $T$ consisting of the identity cosets of vertex and edge groups of $\Psi$ is a transversal of $T$, a subtree of $T$, and the stabilizers of the vertices and edges of the transversal are simply the corresponding vertex and edge groups of $\Psi$. If $s \in S$ belongs to two vertex groups, then $\psi^{-1}(s)$ stabilizes the corresponding vertices of the transversal, hence stabilizes all the edges and vertices in a geodesic path in $T$ between these vertices, and so $s$ belongs to all the vertex and edge groups in the path in $\Psi$ between these vertices. Hence the vertices and edges with groups containing $s$ form a subtree in $\Psi$. If there is an edge in $\Gamma(W, S)$ between $s, t \in S$, then the subgroup $U$ of $W$ generated by $s$ and $t$ is finite. But then $\psi^{-1}(U)$ is a finite subgroup of $\pi_{1}(\Psi)$ acting on $T$ and so must stabilize a vertex $V$ of $T$. Let $V^{\prime}$ 
be the vertex of the transversal closest to $V$ in $T$. Then $V^{\prime}$ lies between $V$ and a vertex of the transversal stabilized by $s$ and so is also stabilized by $s$, and similarly for $t$. Thus $V^{\prime}$ corresponds to a vertex of $\Psi$ having vertex group containing both $s$ and $t$ and this special subgroup has an edge between $s$ and $t$ in its presentation diagram as well.

Conversely, suppose $\Psi$ is a graph of groups with each edge of the presentation diagram of $W$ in the presentation diagram of some vertex group, and such that, for each generator $s \in S$, the vertices and edges of $\Psi$ with groups containing $s$ form a subtree in $\Psi$. All of the occurrences of an $s \in S$ in different vertex groups of $\Psi$ are identified by the relators of $\pi_{1}(\Psi)$ along edges in the subtree of vertex and edge groups containing $s$. Take $\psi: \pi_{1}(\Psi) \rightarrow W$ extending the inclusion map of the generators $S$ in $\pi_{1}(\Psi)$ into $W$. We get that $\psi$ is an isomorphism by checking that each of the defining relators of $W$ is already a relator of $\pi_{1}(\Psi)$. But each generator of $W$ belongs to some vertex group of $\Psi$ where it has order 2, and the other relators of $W$ correspond to edges of the presentation diagram and these relators also already hold in some vertex group of $\Psi$.

If $\Psi$ is a visual graph of groups decomposition for the Coxeter system $(W, S)$, it is convenient to label a vertex of $\Psi$ by the subset of $S$ that generates the corresponding vertex group. So if $Q \subset S$ is a vertex label of $\Psi$, then $\Psi(Q)=\langle Q\rangle$. If $\Psi$ is reduced, its vertex labels are distinct and we identify vertices with their labels. Even if $\Psi$ is reduced, two distinct edges may have the same edge group, so we do not extend this labeling to edges.

The following two technical results are useful tools.

Corollary 5. Suppose that $(W, S)$ is a finitely generated Coxeter system, $\Psi$ is a visual graph of groups decomposition of $W$, and $E \subset S$ is such that $\langle E\rangle=\Psi\left(E^{\prime}\right)$ for $E^{\prime}$ an edge of $\Psi$. If $\{x, y\} \subset S-E$, and $x \in X$ and $y \in Y$ for $X$ and $Y$ labels of vertices of $\Psi$ on opposite sides of $E^{\prime}$, then as a subset of $\Gamma(W, S), E$ separates $x$ and $y$ in $\Gamma$.

Proof. Otherwise, among all such $x, y$ that fail the conclusion, let $\alpha$ be a shortest path in $\Gamma$ from $x$ to $y$ avoiding $E$. Note that by Lemma $4, x \neq y$. If $x \equiv x_{0}, x_{1}, \ldots x_{n} \equiv y$ are the consecutive vertices of $\alpha$, then, by Lemma $4,\left\{x_{0}, x_{1}\right\} \subset V$ for some vertex label $V$ of $\Psi$. Since $x_{0} \notin E$, Lemma 4 implies that $V$ and $X$ label vertices on the same side of $E^{\prime}$ in $\Psi$. But then $x_{1}$ and $y$ satisfy the hypothesis of the corollary, contradicting the minimality of $\alpha$.

Corollary 6. Suppose that $(W, S)$ is a finitely generated Coxeter system, and $\Psi$ is a visual graph of groups decomposition of $W$. If $C$ is a complete subset of the presentation diagram $\Gamma(W, S)$, then there is a vertex labeled $V(\subset S)$ of $\Psi$ such that $C \subset V$. 
Proof. We may assume that $\Psi$ is reduced. If $C$ is not a subset of a vertex of $\Psi$, then there is a vertex $U \subset S$ of $\Psi$ containing a maximal number of elements of $C$ and $V \subset S$ a vertex of $\Psi$ closest to $U$ such that $V$ contains an element $c$ of $C-U$. If $E^{\prime}$ is the last edge of the $\Psi$-geodesic from $U$ to $V$ and $\Psi\left(E^{\prime}\right)=\langle E\rangle$ for $E \subset S$, then $(U \cap C) \not \subset E$ and $c \notin E$. By Corollary $5, E$ separates $(U \cap C)-E$ from $c$ in $\Gamma$. This is impossible since $C$ is complete.

\section{Proof of main results}

We begin this section with a proof of Theorem 1 and conclude with a proof of Theorem 2. Several examples are presented to introduce the reader to visual decompositions.

Proof of Theorem 1. Suppose that $(W, S)$ is a Coxeter system and suppose $\Lambda$ is a graph of groups with $W$ a subgroup of $\pi_{1}(\Lambda)$. We may identify the vertex and edge groups of $\Lambda$ with subgroups of $\pi_{1}(\Lambda)$. Then $\pi_{1}(\Lambda)$ acts on the Bass-Serre tree $T$ such that the stabilizers of vertices of the tree are conjugates of the vertex groups of $\Lambda$ and stabilizers of edges of the tree are conjugates of the edge groups of $\Lambda$.

We build a visual graph of groups decomposition $\Psi$ of $W$ with this tree as its graph. For each vertex $V$ (resp. edge $E$ ) of $\Psi$ take vertex group $\Psi(V)$ (resp. edge group $\Psi(E)$ ) to be the subgroup of $W$ generated by the $s \in S$ stabilizing $V$ (resp. $E$ ). The edge groups inject into the vertex groups by inclusion maps. Clearly, each vertex group (resp. edge group) of $\Psi$ is a special subgroup of $W$ and a subgroup of a conjugate of a vertex group (resp. edge group) of $\Lambda$. Each generator $s \in S$ is of order 2 and so stabilizes some vertex of $T$. If two vertices of $T$ are stabilized by $s$, then $s$ stabilizes the geodesic path between the vertices, thus the subgraph of $T$ of vertices and edges stabilized by $s$ is a subtree. Suppose that there is an edge in the presentation diagram for $(W, S)$ between $s$ and $t$ in $S$. The subgroup generated by $s$ and $t$ has finite order, so stabilizes some vertex of $T$, with $s$ and $t$ belonging to that vertex group. By Lemma 4 then, $\pi_{1}(\Psi)$ is isomorphic to $W$ and $\Psi$ is a visual graph of groups decomposition of $W$.

Finally, if $U$ is a special subgroup and a subgroup of a conjugate of one of the vertex groups of $\Lambda$, then $U$ stabilizes a vertex in $T$ and so $U$ is a subgroup of the vertex group of that vertex.

Generally, $\Psi$ will be an infinite tree. If $W$ is finitely generated, by taking the subtree spanning a finite set of vertices whose vertex groups between them include all of the elements of $S$, we get a finite visual graph of groups decomposition of $W$. Reducing this graph of groups results in a reduced visual graph of groups decomposition for $W$. 
Remark 1. If $W, \Lambda$ and $\Psi$ are as in Theorem 1, then each edge $E$ of $\Lambda$ defines a splitting of $W$ as $A * \Lambda(E) B$ where $A$ is generated by the vertex groups of the vertices on one side of $E$ in $\Lambda$ and $B$ is generated by those on the other side of $E$ in $\Lambda$. There is a quotient map from the Bass-Serre tree for $\Lambda$ to that of $A * \Lambda(E) B$ which respects the action of $W$. If $A \neq \Lambda(E) \neq B$ then $W$ is not a subgroup of a conjugate of $A$ or $B$ and the proof above shows that there is an edge of $\Psi$ such that the edge divides $\Psi$ into two components, neither alone having all of the generators of $W$ in its vertex groups, and such that the corresponding edge group lies in a conjugate of $\Lambda(E)$ (and so also after applying possible reductions to $\Psi$ ).

Example 1. As an example of the main theorem consider the Coxeter group $W$ with presentation

$$
P=\left\langle s_{1}, s_{2}, s_{3}, s_{4}, s_{5}: s_{i}^{2},\left(s_{1} s_{2}\right)^{2}=\left(s_{2} s_{3}\right)^{2}=\left(s_{3} s_{4}\right)^{2}=\left(s_{4} s_{5}\right)^{2}=1\right\rangle .
$$

Note that $\left(s_{3} s_{5} s_{3}\right)^{2}=\left(s_{4} s_{3} s_{5} s_{3}\right)^{2}=1$ so the map $\phi: W \rightarrow W$ defined on generators by $\phi\left(s_{i}\right)=s_{i}$ for $i<5$, and $\phi\left(s_{5}\right)=s_{3} s_{5} s_{3}$, extends to a homomorphism, is surjective, is its own inverse, and so is an automorphism of $W$. Consider subgroups $A=\left\langle s_{1}, s_{2}, s_{4}, s_{3} s_{5} s_{3}\right\rangle, B=\left\langle s_{2}, s_{3}, s_{4}\right\rangle$, and $C=\left\langle s_{2}, s_{4}\right\rangle$. Then $W=A *_{C} B$ is the image under $\phi$ of a visual amalgamated product decomposition of $W$, but $A$ is not a visual subgroup of $W$. We refine this decomposition to a visual graph of groups decomposition following the proof of Theorem 1.

The Bass-Serre tree on which $W$ acts has vertices corresponding to the different cosets of $A$ and $B$ and edges corresponding to the different cosets of $C$ with $h C$ connecting $h A$ and $h B$ for each $h \in W$. The vertex $A$ (respectively $B$ ) of this tree has one edge for each coset $a C$ (resp. $b C$ ) of $C$ in $A$ (resp. $B$ ). An element $g \in W$ acts on this tree by mapping $h A$ to $g h A$ and similarly for cosets of $B$ and $C$. The stabilizer of $g A$ is the conjugate $g A g^{-1}$. The graph of groups $\Psi$ is defined on this tree by taking the vertex and edge groups generated by the $s_{i}$ which stabilize that vertex or edge. Thus $s_{1}, s_{2}$, and $s_{4}$ stabilize the $A$ vertex in the tree, $s_{2}$ and $s_{4}$ stabilize both the $C$ edge and the $s_{3} C$ edge, $s_{2}, s_{3}$, and $s_{4}$ stabilize the $B=s_{3} B$ vertex, $s_{2}, s_{4}$, and $s_{5}$ stabilize the $s_{3} A$ vertex (etc.). Since the vertex groups of these three vertices include all of the generators of $W$, the vertex and edge groups for the rest of the tree collapse back to this three vertex subgraph with the same fundamental group as the graph of groups. The visual decomposition reduces to a three factor amalgamated product

$$
W=\left\langle s_{1}, s_{2}, s_{4}\right\rangle *_{C} B *_{C}\left\langle s_{2}, s_{4}, s_{5}\right\rangle,
$$

where the first and third factors are generated by those generators of $W$ stabilizing $A$ and $s_{3} A$, respectively, are subgroups of conjugates of $A$, and are special subgroups of $W$.

This simple example illustrates that an amalgamated product decomposition of a Coxeter group need not refine to a visual amalgamated product decomposition of only 
two factors. Instead the visual graph of groups decomposition we have produced is sometimes necessary.

Proof of Theorem 2. Let $\Phi_{V}$ be the decomposition of $\Lambda(V)$ given by selecting a fundamental transversal for its action on $T_{\Psi}$, the Bass-Serre tree for $\Psi$. That is, take the graph of $\Phi_{V}$ to be a subtree of $T_{\Psi}$ containing a single vertex and edge from each orbit of the action of $\Lambda(V)$ on $T_{\Psi}$ and take the group of a vertex or edge to be the subgroup of $\Lambda(V)$ stabilizing that vertex or edge. If $U$ is a vertex of $\Phi_{V}$, then $U \in T_{\Psi}$ corresponds to a coset $g \Psi(X)$ of a vertex group $\Psi(X)$ of $\Psi$. The stabilizer in $W$ of $U$ is simply the conjugate $g \Psi(X) g^{-1}$, and so $\Phi_{V}(U)=\Lambda(V) \cap\left(g \Psi(X) g^{-1}\right)$. The group $\Psi(X)$ is a subgroup of a conjugate $h \Lambda(Y) h^{-1}$ of a vertex group $\Lambda(Y)$ of $\Lambda$. Hence $\Phi_{V}(U)$ is a subgroup of $\Lambda(V)$ and of a conjugate $g h \Lambda(Y) h^{-1} g^{-1}$ of $\Lambda(Y)$, and so stabilizes the vertices of $T_{\Lambda}$ corresponding to $\Lambda(V)$ and $g h \Lambda(Y)$. If $V=Y$ and $g h \in \Lambda(V)$, then these are the same vertex of $T_{\Lambda}$, and $g \Psi(X) g^{-1} \subset$ $g h \Lambda(Y) h^{-1} g^{-1}=\Lambda(V)$. Thus $\Phi_{V}(U)=\Lambda(V) \cap\left(g \Psi(X) g^{-1}\right)=g \Psi(X) g^{-1}$ is equal to a conjugate of a vertex group of $\Psi$. Otherwise, $\Lambda(V)$ and $g h \Lambda(Y)$ are different vertices of $T_{\Lambda}$. The group $\Phi_{V}(U)$ stabilizes both vertices and so a geodesic path between the two. In particular, $\Phi_{V}(U)$ stabilizes the first edge $k \Lambda(E)$ in this path, where $E$ is an edge of $\Lambda$ incident with $V$ and $k \in \Lambda(V)$. Thus $\Phi_{V}(U) \subset k \Lambda(E) k^{-1}$. Reducing $\Phi_{V}$ gives the desired graph of groups decomposition.

The next corollary and example examine technical aspects of the decompositions of Theorem 2 .

Corollary 7. Suppose that $(W, S)$ is a finitely generated Coxeter system, $\Lambda$ is a graph of groups decomposition of $W, \Psi$ is a reduced $(W, S)$-visual decomposition from $\Lambda$ and $\Phi_{V}$ is a reduced graph of groups decomposition for the vertex group $\Lambda(V)$ as given in the proof of Theorem 2. If $X$ is a vertex of $\Psi, V$ is a vertex of $\Lambda$, and $g \Psi(X) g^{-1}$ is a subgroup of $\Lambda(V)$ for some $g \in W$, then $\operatorname{vg} \Psi(X) g^{-1} v^{-1}$ is a vertex group of $\Phi_{V}$ for some $v \in \Lambda(V)$.

Proof. Let $\Phi_{V}^{\prime}$ be the decomposition of $\Lambda(V)$ given by selecting a fundamental transversal for its action on $T_{\Psi}$, the Bass-Serre tree for $\Psi$, and let $\Phi_{V}$ be reduced from $\Phi_{V}^{\prime}$. By the definition of transversal, there is $v \in \Lambda(V)$ such that the coset $v g \Psi(X)$ corresponds to a vertex $B$ of the transversal. Then $\Phi_{V}^{\prime}(B)=\Lambda(V) \cap$ $v g \Psi(X) g^{-1} v^{-1}=v g \Psi(X) g^{-1} v^{-1}$. It remains to show that this vertex group survives reduction. Otherwise, there is a vertex $Q$ of $\Phi_{V}^{\prime}$ such that $v g \Psi(X) g^{-1} v^{-1}$ is a proper subgroup of $\Phi_{V}^{\prime}(Q) \equiv \Lambda(V) \cap h \Psi(Z) h^{-1}$ (where $Q \in T_{\Psi}$ corresponds to the coset $h \Psi(Z))$. But then $\Psi(X)$ is a proper subgroup of $g^{-1} v^{-1} h \Psi(Z) h^{-1} v g$ which is impossible by Lemma 3.

The next example shows that the previous corollary cannot be extended to show that a vertex group of $\Psi$ is conjugate to a vertex group of $\Phi_{V}$ for a unique vertex $V$ 
of $\Lambda$. In fact, all vertex groups of a particular $\Phi_{V}$ may be equal to vertex groups of other $\Phi_{Q}$ for $Q$ a vertex of $\Lambda$.

Example 2. Consider the group $W$ with Coxeter presentation $\left\langle w, x, y, z: w^{2}=\right.$ $\left.x^{2}=y^{2}=z^{2}=1\right\rangle$ (and free product decomposition $\langle w\rangle *\langle x\rangle *\langle y\rangle *\langle z\rangle$ ). There is an automorphism of $W$ that fixes $w$ and $y$, and maps $x$ to wyxyw and $z$ to $y(w y x y w) z(w y x y w) y$. $W$ has the decomposition $\langle w, x\rangle *\langle x\rangle\langle x, z\rangle *\langle z\rangle\langle y, z\rangle$. Hence the automorphism induces a decomposition $\Lambda$, with vertices $V_{1}$ and $V_{2}$ and $V_{3}$, where $V_{1}$ and $V_{2}$ are connected by an edge $E_{1}$, and $V_{2}$ and $V_{3}$ are connected by an edge $E_{2}$. The decomposition $\Lambda \equiv \Lambda\left(V_{1}\right) *_{\Lambda\left(E_{1}\right)} \Lambda\left(V_{2}\right) *_{\Lambda\left(E_{2}\right)} \Lambda\left(V_{3}\right)$ of $W$ is such that

$$
\begin{aligned}
\Lambda\left(V_{1}\right) & =\langle w, w y x y w\rangle, \\
\Lambda\left(E_{1}\right) & =\langle w y x y w\rangle, \\
\Lambda\left(V_{2}\right) & =\langle w y x y w, y(w y x y w) z(w y x y w) y\rangle, \\
\Lambda\left(E_{2}\right) & =\langle y(w y x y w) z(w y x y w) y\rangle, \\
\Lambda\left(V_{3}\right) & =\langle y, y(w y x y w) z(w y x y w) y\rangle .
\end{aligned}
$$

A visual decomposition $\Psi$, from $\Lambda$, is $\langle w\rangle *\langle x\rangle *\langle y\rangle *\langle z\rangle$. The decomposition $\Phi_{V_{1}}$ of Theorem 2 is $\langle w\rangle *\langle w y x y w\rangle, \Phi_{V_{2}}$ is $\langle w y x y w\rangle *\langle y(w y x y w) z(w y x y w) y\rangle$, and $\Phi_{V_{3}}$ is $\langle y\rangle *\langle y(w y x y w) z(w y x y w) y\rangle$. Hence the vertex groups $\langle x\rangle$ and $\langle z\rangle$ of $\Psi$ are conjugate to vertex groups of $\Phi_{V}$ for more than one vertex $V$ of $\Lambda$. Furthermore, both vertex groups of $\Phi_{V_{2}}$ are equal to vertex groups of other $\Phi_{V_{i}}$.

Example 3. Consider the Coxeter presentation $\left\langle a, b, c: a^{2}=b^{2}=c^{2}=1\right\rangle$. This group splits as $\Lambda \equiv\langle a, b c\rangle *\langle b c\rangle\langle b, c\rangle$. The visual decomposition from this splitting is $\Psi \equiv\langle a\rangle *\langle b, c\rangle$. If $\Lambda(V)$ is the vertex group $\langle a, b c\rangle$, then its graph of groups decomposition $\Phi_{V}$, induced by its action on $T_{\Psi}$, the Bass-Serre tree for $\Psi$, is $\mathbb{Z}_{2} * \mathbb{Z}$. This group also has a decomposition with underlying graph not a tree, but it is not induced by its action on $T_{\Psi}$. We wonder if every decomposition of a finitely generated Coxeter group and corresponding visual decomposition can only induce $\Phi_{V}$ having as underlying graph a tree.

In their accessibility paper [2], Bestvina and Feighn limit the number of vertex groups that can occur in a reduced graph of groups decomposition with "small" edge groups, for a given finitely presented group. Strong accessibility results for Coxeter groups over "minimal" splittings are the focus of [14]. While Theorem 2 limits how far a vertex group of an arbitrary decomposition of a Coxeter group can stray from visual, the following example shows that one cannot expect an accessibility result for Coxeter groups over arbitrary splittings.

Example 4. For a virtually free (non-virtually cyclic) group, the following technique is standard for creating non-trivial reduced graph of groups decompositions with $n$ 
vertex groups for any $n \in\{1,2, \ldots\}$. Let $W$ be the Coxeter group with presentation $\left\langle s_{1}, s_{2}, s_{3}, s_{4}, s_{5}: s_{i}^{2}=1\right\rangle$. Then $W$ has free product decomposition $A * B$ where $A=\left\langle s_{1}, s_{2}, s_{3}\right\rangle$ and $B=\left\langle s_{4}, s_{5}\right\rangle$. The element $b \equiv s_{4} s_{5}$ generates an infinite cyclic subgroup of finite index in $B$ and $A$ contains a non-abelian free subgroup of finite index. Let $\left\{a_{1}, a_{2}, \ldots\right\}$ be a free generating set for an infinite rank free subgroup of $A$. Then $W$ has the decomposition

$$
\Lambda_{1}=\langle A \cup\{b\}\rangle *\left\langle a_{1}, b\right\rangle\left\langle B \cup\left\{a_{1}\right\}\right\rangle .
$$

The group $\langle A \cup\{b\}\rangle$ has the following non-trivial reduced graph of groups decomposition, which is compatible with $\Lambda_{1}$ :

$$
\left\langle A \cup\left\{b^{2}\right\}\right\rangle *_{\left\langle a_{1}, a_{2}, b^{2}\right\rangle}\left\langle a_{1}, a_{2}, b\right\rangle .
$$

Hence $W$ has the reduced decomposition

$$
\Lambda_{2}=\left\langle A \cup\left\{b^{2}\right\}\right\rangle *\left\langle a_{1}, a_{2}, b^{2}\right\rangle\left\langle a_{1}, a_{2}, b\right\rangle *\left\langle a_{1}, b\right\rangle\left\langle B \cup\left\{a_{1}\right\}\right\rangle .
$$

Let $E_{n}=\left\langle a_{1}, \ldots, a_{n}, b^{\left(2^{n-2}\right)}\right\rangle$, let $C_{n}=\left\langle a_{1}, \ldots, a_{n}, b^{\left(2^{n-1}\right)}\right\rangle$, and let $A_{n}=\left\langle A \cup\left\{b^{\left(2^{n-1}\right)}\right\}\right\rangle$. Then we have a reduced decomposition for $W$ with $n+1$ vertex groups:

$$
\Lambda_{n}=A_{n} * C_{n} E_{n} * C_{n-1} E_{n-1} * C_{n-2} * \cdots * E_{2} * C_{1}\left\langle B \cup\left\{a_{1}\right\}\right\rangle .
$$

For each $n, A * B$ is a visual decomposition from $\Lambda_{n}$.

\section{Technical results}

As mentioned in the introduction, our basic reference for Coxeter groups is [3]. The technical results of this section are well known and can be derived from two fundamental facts: the first concerning special subgroups of Coxeter groups and the second called the "deletion condition".

Proposition 8. Suppose that $(W, S)$ is a Coxeter system and $P=\left\langle S:(s t)^{m(s, t)}\right.$ for $m(s, t)<\infty\rangle$ (where $m: S^{2} \rightarrow\{1,2, \ldots, \infty\}$ ) is a Coxeter presentation for $W$. If $A \subset S$, then $(\langle A\rangle, A)$ is a Coxeter system with Coxeter presentation $\left\langle A:(s t)^{m^{\prime}(s, t)}\right.$ for $\left.m^{\prime}(s, t)<\infty\right\rangle$ (where $\left.m^{\prime}=\left.m\right|_{A^{2}}\right)$.

Given a group $G$ and a generating set $S$, an $S$-geodesic for $g \in G$ is a shortest word in $S \cup S^{-1}$ such that the product of the letters of this word is $g$. The number of letters in an $S$-geodesic for $g$ is the $S$-length of $g$. We include an elementary proof of the deletion condition pointed out to us by A. Yu. Ol'shanskii and based on the theory of van Kampen diagrams (see Chapter 5 of Lyndon and Schupp's book [13] for a basic introduction to van Kampen diagrams). 
Proposition 9 (The deletion condition). Suppose that $(W, S)$ is a Coxeter system and $w=a_{1} \ldots a_{n}$ for $a_{i} \in S$. If $a_{1} \ldots a_{n}$ is not geodesic then there are indices $i<j$ in $\{1,2, \ldots, n\}$ such that $w=a_{1} \ldots a_{i-1} a_{i+1} \ldots a_{j-1} a_{j+1} \ldots a_{n}$, i.e., $a_{i}$ and $a_{j}$ can be deleted.

Proof. Let $b_{1} \ldots b_{m}$ be an $S$-geodesic for $w$, so that $m<n$. Let $\mathscr{D}$ be a van Kampen diagram for the word $a_{1} \ldots a_{n} b_{m} \ldots b_{1}$. The relation 2-cell $r_{1}$ containing $a_{1}$ is of even length and so there is an edge $e_{1}$ of $r_{1}$ "opposite" $a_{1}$. The two subpaths of the boundary of $r_{1}$ separated by $a_{1}$ and $e_{1}$ have the exact same labeling. Let $r_{2}$ be the relation 2-cell of $\mathscr{D}$ that shares $e_{1}$ with $r_{1}$, and let $e_{2}$ be the edge of $r_{2}$ opposite $e_{1}$. Again the labeling of the two subpaths of the boundary of $r_{2}$ separated by $e_{1}$ and $e_{2}$ are the same. Continue until $e_{k}$ is on the boundary of $\mathscr{D}$. The "strip" determined by $\bigcup_{i=1}^{k} r_{i}$ is such that the two subpaths of the boundary of this strip separated by $a_{1}$ and $e_{k}$ have the same labeling. This creates a unique paring of edges on the boundary of $\mathscr{D}$. Since $m<n$ there are indices $i<j$ in $\{1,2, \ldots, n\}$ such that $a_{i}$ is paired with $a_{j}$. If the two subpaths of the boundary of the strip for $a_{i}$ separated by $a_{i}$ and $a_{j}$ are labeled $\beta$, then (as the product of edge path labels of loops in $\mathscr{D}$ is trivial in $W$ ) $a_{i+1} \ldots a_{j-1}=\beta=a_{i} \ldots a_{j}$, i.e., $a_{i}$ and $a_{j}$ are deleted.

Lemma 10. Suppose that $(W, S)$ is a Coxeter system, $A \subset S$, and $a \in\langle A\rangle$. If $a=a_{1} \ldots a_{n}=b_{1} \ldots b_{n}$ are $S$-geodesics then $\left\{a_{1}, \ldots, a_{n}\right\}=\left\{b_{1}, \ldots, b_{n}\right\}$.

Proof. Assume $n$ is minimal among all counterexamples to the lemma. By Proposition 8 , we have $n \neq 1$. Note that $a_{1} \ldots a_{n} b_{n}=b_{1} \ldots b_{n-1}$. By the deletion condition, $b_{n}$ is deleted together with some $a_{i}$ in the first expression. Inductively it follows that $\left\{b_{1}, \ldots, b_{n-1}\right\} \subset\left\{a_{1}, \ldots, a_{n}\right\}$. Similarly, $b_{1} a_{1} \ldots a_{n}=b_{2} \ldots b_{n}$ so that $\left\{b_{2}, \ldots, b_{n}\right\} \subset\left\{a_{1}, \ldots, a_{n}\right\}$.

If $G$ is a group with generating set $S$, then the Cayley graph $\mathcal{K}(G, S)$ is a labeled graph with vertex set $G$ and a directed edge (labeled $s$ ) from the vertex $g$ to the vertex $g s$ for each $s \in S$. Given a vertex $x$ of $\mathcal{K}(G, S)$, there is a bijective correspondence between edge paths at $x$ and words in $S \cup S^{-1}$, where traversing an edge labeled $s$ opposite its orientation is read as $s^{-1}$. Hence $S$-geodesics and $\mathcal{K}(G, S)$-geodesics at a given vertex are the same.

The next result is a straightforward application of the deletion condition.

Lemma 11. Suppose that $(W, S)$ is a Coxeter system, $\{x, y\} \subset W$ and $A \subset S$. Then in the Cayley graph $\mathcal{K}(W, S)$ there is a unique closest point $z$ of the coset $y\langle A\rangle$ to $x$. Furthermore, if $\alpha$ is the $S$-geodesic from $x$ to $z$, and $\beta$ is any $A$-geodesic at $z$, then $\alpha \beta$ is an $S$-geodesic.

The next lemma follows by a result of Kilmoyer (see Theorem 2.7.4 of [6]), but we include a direct proof for completeness. 
Lemma 12. Suppose that $(W, S)$ is a Coxeter system, $I, J \subseteq S$, and $d$ is a minimal length double coset representative in $\langle I\rangle d\langle J\rangle$. Then $\langle I\rangle \cap d\langle J\rangle d^{-1}=\langle K\rangle$ where $K=I \cap\left(d J d^{-1}\right)$. Hence $g\langle I\rangle g^{-1} \cap h\langle J\rangle h^{-1}=f\langle K\rangle f^{-1}$ for $K \subset I$.

Proof. With $K=I \cap\left(d J d^{-1}\right)$, clearly, $\langle K\rangle \subset\langle I\rangle \cap d\langle J\rangle d^{-1}$. Suppose that $a$ is a shortest element in $\langle I\rangle \cap d\langle J\rangle d^{-1}$ but not in $\langle K\rangle$. Write $a=a_{1} a_{2} \ldots a_{m}$ geodesically with $a_{i} \in I$ and $d^{-1} a d=b_{1} b_{2} \ldots b_{n}$ geodesically for $b_{i} \in J$. Write $d=d_{1} d_{2} \ldots d_{k}$ geodesically for $d_{i} \in S$. Then since $d$ is a minimal length double coset representative, $a d=a_{1} a_{2} \ldots a_{m} d_{1} d_{2} \ldots d_{k}=d_{1} d_{2} \ldots d_{k} b_{1} b_{2} \ldots b_{n}$ are each geodesic. Hence $m=n$. Clearly $a \neq 1$, and $a \notin S$ else $a=a_{1}=d b_{1} d^{-1} \in K$ by definition. Instead, $m>1$. Now $b_{1} b_{2} \ldots b_{m} d^{-1}$ and $d^{-1} a_{m}$ are geodesic, but $d^{-1} a_{1} \ldots a_{m-1}=b_{1} b_{2} \ldots b_{m} d^{-1} a_{m}$, so this last is not geodesic and, by the deletion condition, $a_{m}$ is deleted together with some $b_{i}$ to give $b_{1} b_{2} \ldots b_{m} d^{-1} a_{m}=$ $b_{1} \ldots b_{i-1} b_{i+1} \ldots b_{m} d^{-1}$ (if $1<i<m$ and similarly if $i=1$ or $i=m$ ). But then $a_{1} a_{2} \ldots a_{m-1}=d b_{1} \ldots b_{i-1} b_{i+1} \ldots b_{m} d^{-1} \in\langle I\rangle \cap d\langle J\rangle d^{-1}$ and, by the minimality of $a$ and Lemma 10 , we have $\left\{a_{1}, a_{2}, \ldots, a_{m-1}\right\} \subset K$. Likewise $\left\{a_{m}, \ldots, a_{2}\right\} \subset\langle I\rangle \cap d\langle J\rangle d^{-1}$, so $a_{m} \in K$. But then $a \in\langle K\rangle$, contradicting the choice of $a$. Instead, every $a \in\langle I\rangle \cap d\langle J\rangle d^{-1}$ is in $\langle K\rangle$.

Given conjugates $g\langle I\rangle g^{-1}$ and $h\langle J\rangle h^{-1}$ of special subgroups, take $d$ of minimal length in $\langle I\rangle g^{-1} h\langle J\rangle$, so $g^{-1} h=a d b^{-1}$ for $a \in\langle I\rangle$ and $b \in\langle J\rangle,\langle I\rangle g^{-1} h\langle J\rangle=$ $\langle I\rangle d\langle J\rangle$, and $\langle I\rangle \cap d\langle J\rangle d^{-1}=\langle K\rangle$ as above. Then

$$
\begin{aligned}
g\langle I\rangle g^{-1} \cap h\langle J\rangle h^{-1} & =g a\langle I\rangle a^{-1} g^{-1} \cap h b\langle J\rangle b^{-1} h^{-1} \\
& =g a\left(\langle I\rangle \cap d\langle J\rangle d^{-1}\right) a^{-1} g^{-1} \\
& =g a\langle K\rangle a^{-1} g^{-1} .
\end{aligned}
$$

We will use the following corollary in Section 6 in our analysis of FA subgroups.

Corollary 13. Suppose that $(W, S)$ is a finitely generated Coxeter system, $I, J \subseteq S$, the induced subgraph on $I$ is a maximal complete subgraph of $\Gamma(W, S)$, and $I \subseteq$ $w\langle J\rangle w^{-1}$ for some $w \in W$. Then $I \subseteq J$ and $w \in\langle J\rangle$.

Proof. Take $d$ a minimal length double coset representative in $\langle I\rangle w\langle J\rangle$ so $w=$ $a d b^{-1}$ for $a \in\langle I\rangle$ and $b \in\langle J\rangle$. Then since

$$
\begin{aligned}
\langle I\rangle \cap d\langle J\rangle d^{-1} & =a^{-1}\left(a\langle I\rangle a^{-1} \cap a d b^{-1}\langle J\rangle b d^{-1} a^{-1}\right) a \\
& =a^{-1}\left(\langle I\rangle \cap w\langle J\rangle w^{-1}\right) a \\
& =\langle I\rangle,
\end{aligned}
$$

we have $I \subseteq d J d^{-1}$ and so $d^{-1} I d \subseteq J$. If $d \neq 1$ and $d=d_{1} d_{2} \ldots d_{k}$ geodesically, then by the minimality of $d, d_{1} \notin I$. There is some $s \in I$ unrelated to $d_{1}$, since $I$ induces a maximal complete subgraph of the Coxeter diagram. But if $a_{1} \ldots a_{k}$ is 
any geodesic and $s$ is unrelated to $a_{k}$ then $a_{1} \ldots a_{k} s$ is geodesic (apply Lemma 11 with $x=1, y=a_{1} \ldots a_{k-1}, A=\left\{a_{k}, s\right\}$ and $\beta=\beta_{1} a_{k} s$, where $\beta_{1}$ is the $\left\{a_{k}, s\right\}$ geodesic from $z$ to $y$ ). Hence $d^{-1} s d$ cannot have length 1 and cannot be in $J$. Instead then, $d=1, I \subseteq J$ and $w=a d b^{-1} \in\langle J\rangle$.

The following corollaries find application in the analysis of rigidity of Coxeter groups.

Corollary 14. If $(W, S)$ is a finitely generated Coxeter system, $I \subseteq S$, and $w^{-1}\langle I\rangle w \subset\langle I\rangle$ for some $w \in W$, then $w^{-1}\langle I\rangle w=\langle I\rangle$.

Proof. Let $d$ be a minimal length double coset representative in $\langle I\rangle w\langle I\rangle$, so $w=$ $a d b^{-1}$ for $a, b \in\langle I\rangle$. Then

$$
\langle I\rangle=\langle I\rangle \cap w\langle I\rangle w^{-1}=a\left(\langle I\rangle \cap d\langle I\rangle d^{-1}\right) a^{-1} .
$$

Hence $\langle I\rangle=\langle I\rangle \cap d\langle I\rangle d^{-1}=\left\langle I \cap d I d^{-1}\right\rangle$, by Lemma 12. By Propositions 8 and 9, a proper subset of $I$ cannot generate the same Coxeter group as $I$, so $I=d I d^{-1}$. Hence $d^{-1} I d=I$ and $w^{-1}\langle I\rangle w=b d^{-1}\langle I\rangle d b^{-1}=b\langle I\rangle b^{-1}=\langle I\rangle$.

Corollary 15. Suppose that $(W, S)$ and $\left(W, S^{\prime}\right)$ are finitely generated Coxeter systems for the same Coxeter group $W$. Suppose that $I \subset S$ is such that the induced subgraph of I separates $\Gamma(W, S)$ (its complement has at least two components). Then there are sets $J \subset S$ and $J^{\prime} \subset S^{\prime}$ such that the induced subgraphs of $J$ and $J^{\prime}$ separate $\Gamma(W, S)$ and $\Gamma\left(W, S^{\prime}\right)$, respectively, $\langle J\rangle$ and $\left\langle J^{\prime}\right\rangle$ are conjugate, and $\langle J\rangle$ is conjugate to a subgroup of $\langle I\rangle$.

Proof. As noted in the introduction, since $I$ separates $\Gamma(W, S), W=A *_{\langle I\rangle} B$, for $A$ the $(S)$ special subgroup generated by $I$ and the generators in some components of the complement of $I$, and $B$ the $(S)$ special subgroup generated by $I$ and the generators in the other components of the complement of $I$ in $\Gamma(W, S), A \neq\langle I\rangle \neq B$. Taking this amalgamated product decomposition of $W$ to be $\Lambda$, we consider a corresponding visual decomposition $\Psi$ with respect to the alternate generating set $S^{\prime}$. As noted in Remark 1, there must be an edge of $\Psi$, with not all of the generators $S^{\prime}$ appearing on one side, having edge group $\left\langle I_{1}^{\prime}\right\rangle$, for an $I_{1}^{\prime} \subset S^{\prime}$, which is a subgroup of a conjugate of $\langle I\rangle$. That is, there is an $I_{1}^{\prime} \subset S^{\prime}$ which separates $\Gamma\left(W, S^{\prime}\right)$ and for which $\left\langle I_{1}^{\prime}\right\rangle$ is a subset of a conjugate of $\langle I\rangle$.

But then $I_{1}^{\prime}$ gives a proper splitting of $W$ and by the same reasoning there is an $I_{2} \subset S$ which separates $\Gamma(W, S)$ and which is a subset of a conjugate of $\left\langle I_{1}^{\prime}\right\rangle$. Continuing in this fashion, we can find $I_{2 k+1}^{\prime} \subset S^{\prime}$ separating $\Gamma\left(W, S^{\prime}\right)$, generating a subgroup of a conjugate of $\left\langle I_{2 k}\right\rangle$, and an $I_{2 k+2} \subset S$ separating $\Gamma(W, S)$ and generating a subgroup of a conjugate of $\left\langle I_{2 k+1}^{\prime}\right\rangle$. 
Since there are only finitely many subsets of $S$ or $S^{\prime}$, for some $k_{1}<k_{2}<k_{3}$, $I_{k_{1}}^{\prime}=I_{k_{3}}^{\prime}$. Take $J^{\prime}=I_{k_{1}}^{\prime}=I_{k_{3}}^{\prime}$ and $J=I_{k_{2}}$. Since $\left\langle I_{j}\right\rangle$ and $\left\langle I_{j+1}^{\prime}\right\rangle$ are subgroups of conjugates of $\left\langle I_{i}\right\rangle$ and $\left\langle I_{i+1}^{\prime}\right\rangle$ for $i<j$, take $a_{1}, a_{2} \in W$ such that

$$
\left\langle I_{k_{3}}^{\prime}\right\rangle \subset a_{2}\left\langle I_{k_{2}}\right\rangle a_{2}^{-1} \subset a_{2} a_{1}\left\langle I_{k_{1}}^{\prime}\right\rangle a_{1}^{-1} a_{2}^{-1} .
$$

Then $a_{2}^{-1} a_{1}^{-1}\left\langle J^{\prime}\right\rangle a_{1} a_{2} \subset\left\langle J^{\prime}\right\rangle$, so by Corollary 14 these are equal, and we get

$$
\left\langle J^{\prime}\right\rangle \subset a_{2}\langle J\rangle a_{2}^{-1} \subset a_{2} a_{1}\left\langle J^{\prime}\right\rangle a_{1}^{-1} a_{2}^{-1}=\left\langle J^{\prime}\right\rangle .
$$

Thus $\left\langle J^{\prime}\right\rangle=a_{2}\langle J\rangle a_{2}^{-1}$, and $J$ and $J^{\prime}$ generate subgroups of conjugates of $\langle I\rangle$ that separate $\Gamma(W, S)$ and $\Gamma\left(W, S^{\prime}\right)$ respectively.

\section{Ends}

Stalling's theorem [18] states that if a finitely generated group has more than one end then it splits nontrivially as an amalgamated product or $\mathrm{HNN}$-extension over a finite group. The following result is then an easy consequence of our main theorem. It can also be obtained from work of M. Davis [8].

Corollary 16. For any finitely generated Coxeter group $W$ with presentation diagram $\Gamma$, the following assertions are equivalent:

(1) $W$ has more than one end.

(2) $W$ decomposes as a nontrivial amalgamated product $A *_{C} B$ where $C$ is finite and $A, B$, and $C$ are special subgroups.

(3) $\Gamma$ contains a complete separating subgraph, the vertices of which generate a finite subgroup of $W$.

Proof. If $W$ is not 1 -ended or finite, then by Stallings' splitting theorem, $W=A *_{C} B$ with $C$ finite (and not as an $\mathrm{HNN}$-extension since an $\mathrm{HNN}$-extension maps onto $\mathbb{Z}$ but a homomorphism of a Coxeter group into $\mathbb{Z}$ must take generators to the identity). Then by Theorem 1, $W$ has a reduced visual graph of groups decomposition in which each edge group is a subgroup of a conjugate of $C$ and so is finite. This decomposition cannot be trivial since each vertex group is a subgroup of a conjugate of $A$ or $B$ neither of which is $W$ in the given nontrivial splitting, and so no vertex group can be $W$. Hence there is at least one edge after reducing $\Psi$ and collapsing the other edges gives $W$ as a visual amalgamated product of special subgroups over a finite special subgroup. The remaining implications are easy.

By Stallings' theorem and Theorem 1, a 2-ended Coxeter group splits as a visual amalgamated product over a finite group which is of index two in each factor. The following result then characterizes 2-ended Coxeter groups. 
Corollary 17. A Coxeter group with system $(W, S)$ and diagram $\Gamma$ is 2-ended iff $\Gamma$ contains a separating subdiagram $\Gamma_{0}$ which is the presentation diagram of a finite group, and $\Gamma-\Gamma_{0}$ consists of two vertices each of which is connected to each vertex of $\Gamma_{0}$ by edges labeled 2 (but not connected to each other). Equivalently, $W=\langle x, y\rangle \times\langle H\rangle$ where $\{x, y\} \cup H=S, x$ and $y$ are unrelated and $\langle H\rangle$ is finite.

Thus the number of ends of a Coxeter group can be easily determined from an analysis of separating subdiagrams of a presentation diagram, and checking which subdiagrams correspond to finite subgroups. A Coxeter group whose presentation diagram is complete is either finite or 1-ended. The finite Coxeter groups have been enumerated [3].

A Dunwoody decomposition of a finitely presented group is a graph of groups decomposition of the group with finite edge groups and 1-ended and finite vertex groups. In [10], Dunwoody shows that any finitely presented group has such a decomposition.

Corollary 18. Suppose that $(W, S)$ is a finitely generated Coxeter system and $W$ is the fundamental group of a graph of groups $\Lambda$ where each edge group is finite. Then $W$ has a visual decomposition $\Psi$ where each vertex group is 1-ended or finite and a subgroup of a conjugate of a vertex group of $\Lambda$, and where each edge group is finite.

Proof. Take $\Psi$ the reduced visual graph of groups from $\Lambda$ as given by Theorem 1 . Then each edge group of $\Psi$ is a subgroup of a conjugate of an edge group of $\Lambda$ and so is finite. Suppose that some vertex $V(\subset S)$ of $\Psi$ is such that $\langle V\rangle$ is not 1-ended or finite. Then $\langle V\rangle$ visually splits nontrivially over a finite subgroup. If $E(\subset S)$ is an edge of $\Psi$ with endpoint $V$, then $E \subset V$ and $\langle E\rangle$ is finite. In particular, $E$ induces a complete subgraph in $\Gamma(W, S)$. By Corollary $6, E$ must be contained in a vertex group of the visual decomposition of $\langle V\rangle$, i.e., the splitting of $\langle V\rangle$ is visually compatible with $\Psi$. Replacing $\langle V\rangle$ in $\Psi$ by this splitting gives a visual graph of groups decomposition with finite edge groups. Since a special vertex group is replaced by special vertex groups with fewer generators, repeating this process eventually must end with a visual graph of groups decomposition having finite edge groups and finite or 1-ended vertex groups.

One might wonder how "visual" a Dunwoody decomposition must be.

Theorem 19. Suppose that $(W, S)$ is a finitely generated Coxeter system. If $\Lambda$ is a reduced Dunwoody graph of groups decomposition of $W$ and $\Psi$ is a reduced visual decomposition for $(W, S)$ such that each edge group of $\Psi$ is finite and each vertex group of $\Psi$ is a subgroup of a conjugate of a vertex group of $\Lambda$ (in particular if $\Psi$ is a reduced visual graph of groups decomposition from $\Lambda$ ), then

(1) $\Psi$ is a Dunwoody decomposition; 
(2) there is a (unique) bijection $\alpha$ of the vertices of $\Lambda$ to the vertices of $\Psi$ such that for each vertex $V$ of $\Lambda, \Lambda(V)$ is conjugate to $\Psi(\alpha(V))$;

(3) each edge group of $\Lambda$ is conjugate to a special subgroup for $(W, S)$.

Proof. If $\Phi$ is a graph of groups decomposition of $W$ with finite edge groups, then any finite or 1-ended subgroup of $W$ is a subgroup of a conjugate of a vertex group of $\Phi$ (otherwise, the action of this group on the Bass-Serre tree for $\Phi$ would induce a non-trivial splitting over a finite group). Hence each vertex group of $\Lambda$ is a subgroup of a conjugate of a vertex group of $\Psi$. If a vertex group $A=\Lambda(V)$ of $\Lambda$ is a subgroup of a conjugate of $\Psi\left(V^{\prime}\right)$ for $V^{\prime}$ a vertex of $\Psi$, then since $\Psi\left(V^{\prime}\right)$ is a subgroup of a conjugate of a vertex group of $\Lambda, A$ is a subgroup of a conjugate of a vertex group of a vertex $V^{\prime \prime}$ of $\Lambda$. As noted in Lemma 3, in a reduced graph of groups, the vertex group $A$ at $V$ is a subgroup of a conjugate of a vertex group at $V^{\prime \prime}$ only if $V=V^{\prime \prime}$ and the conjugate is by an element of $A$. But then $A$ is conjugate to $\Psi\left(V^{\prime}\right)$. Again, since no vertex group of $\Psi$ is contained in a conjugate of another, $V^{\prime}$ is uniquely determined, and we set $\alpha(V)=V^{\prime}$. Since each vertex group $\Psi\left(V^{\prime}\right)$ is contained in a conjugate of some $\Lambda(V)$ which is in turn conjugate to $\Psi(\alpha(V))$, we must have $V^{\prime}=\alpha(V)$ and each $V^{\prime}$ is in the image of $\alpha$. In particular, each vertex group of $\Psi$ is 1-ended or finite and so $\Psi$ is a Dunwoody decomposition of $W$.

Since $\Lambda$ is a tree, we can take each edge group of $\Lambda$ as contained in its endpoint vertex groups taken as subgroups of $W$. Hence each edge group is simply the intersection of its adjacent vertex groups (up to conjugation). Since vertex groups of $\Lambda$ correspond to conjugates of vertex groups in $\Psi$, their intersection is conjugate to a special subgroup by Lemma 12.

In Example 1 we have a visual Dunwoody decomposition

$$
\left\langle s_{1}, s_{2}\right\rangle *\left\langle s_{2}\right\rangle\left\langle s_{2}, s_{3}\right\rangle *\left\langle s_{3}\right\rangle\left\langle s_{3}, s_{4}\right\rangle *\left\langle s_{4}\right\rangle\left\langle s_{4}, s_{5}\right\rangle,
$$

which is carried by the automorphism $\phi$ to the Dunwoody decomposition where the last factor is replaced by $\left\langle s_{4}, s_{3} s_{5} s_{3}\right\rangle$. Thus in this theorem we cannot expect a single element to conjugate all factors of a Dunwoody decomposition to the corresponding factors of the corresponding visual decomposition. The connection between an arbitrary Dunwoody decomposition and a visual Dunwoody decomposition is however clearly quite close.

It is worthwhile to see how this analysis of Coxeter groups leads to an understanding of why finitely generated Coxeter groups are accessible. While this argument only re-proves a special case of Dunwoody's accessibility theorem, it is the base case of the main theorems of our papers [14] and [15], where we prove a strong accessibility result for Coxeter groups and splittings over "minimal" splitting subgroups, and a JSJ result for Coxeter groups and splittings over virtually abelian subgroups. 
Lemma 20. Suppose that $(W, S)$ is a finitely generated Coxeter system and $\Lambda$ is a graph of groups decomposition of $W$ with finite edge groups. Suppose that a vertex group of $\Lambda$ splits nontrivially as $A *_{C} B$ over a finite $C$. Then there is a special subgroup or a subgroup of a finite special subgroup of $W$ contained in a conjugate of $B$ which is not also contained in a conjugate of $A$ (and then also with $A$ and $B$ reversed).

Proof. Let $\Lambda^{\prime}$ be the graph of groups resulting from replacing the vertex whose group splits by the graph corresponding to $A *_{C} B$. Let $\Psi$ be the corresponding visual graph of groups decomposition of $W$ and let $T$ be the Bass-Serre tree for $\Psi$. The intersection of any conjugates of $A$ and $B$, or the intersection of distinct conjugates of $B$, is contained in a conjugate of an edge group and so is finite. If an infinite vertex group of $\Psi$ lies in a conjugate of $B$, it cannot also lie in a conjugate of $A$, so suppose that no infinite vertex group of $\Psi$ lies in a conjugate of $B$. From the action of $B$ on $T$ we get a reduced graph of groups decomposition of $B$ with vertex and edge groups contained in conjugates of vertex and edge groups of $\Psi$, in particular all of the edge groups are finite. If any vertex group $B_{1}$ of this decomposition is infinite, it is contained in a conjugate of an infinite vertex group of $\Psi$ which is in turn contained in a conjugate of a vertex group of $\Lambda^{\prime}$ other than $B$. But $B_{1}$ would then be an infinite subgroup of $B$ and contained in a conjugate of another vertex group of $\Lambda^{\prime}$, which is impossible. Instead, the vertex groups of the decomposition of $B$ are finite and conjugate to subgroups of finite special subgroups. Replace $B$ in $\Lambda^{\prime}$ by this graph of groups decomposition and collapse the edge $C$ if it equals one of the vertex groups of the decomposition of $B$ to get a new graph of groups decomposition $\Lambda^{\prime \prime}$ where $A$ is adjacent to a vertex group $B_{1}$ of the decomposition of $B$ by an edge labeled $C_{1}$, a proper subgroup of $B_{1}$ (either with $C_{1}=C$, if no collapse happens, or with $C_{1}$ an edge group of the decomposition of $B$ ). Now $B_{1}$ is finite, contained in $B$, but $B_{1}$ cannot also be contained in a conjugate of $A$, since otherwise $B_{1}$ would stabilize a path from a coset of $A$ to $B_{1}$ in the Bass-Serre tree for $\Lambda^{\prime \prime}$ and hence stabilize a coset of $C_{1}$, i.e., would be contained in a conjugate of $C_{1}$ which has fewer elements than $B_{1}$.

As mentioned earlier, our next theorem follows from Dunwoody's accessibility theorem, and the proof of this theorem leads to a general approach to more complex accessibility and JSJ results.

Theorem 21. Finitely generated Coxeter groups are accessible.

Proof. Suppose that $(W, S)$ is a finitely generated Coxeter system. There are only finitely many special subgroups of $W$ and finitely many subgroups of finite special subgroups. For $G$ a subgroup of $W$ let $n(G)$ be the number of special subgroups or subgroups of finite special subgroups which are contained in any conjugate of $G$ 
(which includes the trivial group), so $1 \leq n(G) \leq n(W)$. For $\Lambda$ a finite graph of groups decomposition of $W$ let $c(\Lambda)=\left(c_{n(W)}, \ldots, c_{2}, c_{1}\right)$ where $c_{i}$ is the count of vertex groups $G$ of $\Lambda$ with $n(G)=i$. Let $<$ order these $n(W)$-tuples lexicographically, a well ordering of $n(W)$-tuples of nonnegative integers. If $\Lambda$ reduces to $\Lambda^{\prime}$ then clearly no $c_{i}$ increases and some $c_{i}$ must decrease. If a vertex group $G$ of $\Lambda$ splits as $A *_{C} B$ to produce a new $\Lambda^{\prime}$, then every subgroup of a conjugate of $A$ or $B$ is a subgroup of a conjugate of $G$, but, by the last lemma, some special subgroup or subgroup of a finite special subgroup is contained in a conjugate of $B$, and so of $G$, but not in a conjugate of $A$. Hence $n(A)<n(G)$, and similarly $n(B)<n(G)$, and so $c\left(\Lambda^{\prime}\right)<c(\Lambda)$ since $c_{n(G)}$ decreases by 1 in going from $\Lambda$ to $\Lambda^{\prime}$ and the only other components that change are $c_{n(A)}$ and $c_{n(B)}$ which are later in the tuples. Since $<$ is a well ordering, there can be no infinite sequence of graph of group decompositions of $W$ resulting from successive reductions or splittings over finite subgroups, i.e., $W$ is accessible.

\section{Maximal FA subgroups of Coxeter groups}

Let $(W, S)$ be a finitely generated Coxeter system. A set of vertices of a complete subgraph of $\Gamma(W, S)$ is called a simplex of $(W, S)$ and the subgroup of $W$ generated by a simplex of $(W, S)$ is called a simplex group of $(W, S)$. If $V$ is a vertex of a graph $\Gamma$, define $l k(V)$, the link of $V$, to be the set of all vertices $V^{\prime}$ of $\Gamma$ such that $V^{\prime}$ is connected to $V$ by an edge. Define $s t(V)$, the star of $V$, to be $l k(V) \cup\{V\}$.

A group $G$ is called FA if for every tree on which $G$ acts, the set of fixed points of $G$ in the tree is non-empty. In $\S 6$ of [17], we find basic results on FA groups. In particular we find:

Proposition 22. If $G$ is denumerable then $G$ is $F A$ iff $G$ is finitely generated, no quotient of $G$ is isomorphic to the infinite cyclic group and $G$ does not split nontrivially as an amalgamated product.

Proposition 23. If an FA group $G$ is a subgroup of $A *_{C} B$ then $G$ is a subgroup of a conjugate of $A$ or $B$.

Proposition 24. Every simplex subgroup of a Coxeter system is FA.

We have then the following result.

Lemma 25. Suppose that $(W, S)$ is a Coxeter system and $G$ is an FA subgroup of $W$, then $G$ is a subgroup of a conjugate of a simplex subgroup of $(W, S)$. 
Proof. If the presentation diagram $\Gamma(W, S)$ is not complete, choose vertices $s$ and $t$ that are not related. Then $l k(s)$ separates $s$ and $t$ in $\Gamma(W, S)$. Hence

$$
W \cong\langle s t(s)\rangle *\langle l k(s)\rangle\langle S-\{s\}\rangle
$$

By Proposition 23, $G$ is a subgroup of a conjugate of $\langle s t(s)\rangle$ or $\langle S-\{s\}\rangle$. Continue splitting until the conclusion is realized.

The following theorem is the main result of this section. Our proof is based in group actions on trees. A more combinatorial approach works equally well.

Theorem 26. The maximal FA subgroups of a Coxeter group $W$ are precisely the conjugates of the special subgroups whose diagrams are the maximal complete subdiagrams of a (and equivalently any) presentation diagram for $W$.

Proof. We have that simplex subgroups are FA and any FA subgroup is contained in a conjugate of a simplex subgroup. If $A$ is a maximal simplex subgroup contained in $w B w^{-1}$ for $B$ another simplex subgroup, then by Corollary $13, A=B$ and $w \in B$.

By a result of Tits [3], up to conjugacy there are only finitely many elements of order 2 in a finitely generated Coxeter group. Hence we have:

Proposition 27. Suppose that $(W, S)$ is a finitely generated Coxeter system. Then the subgroup of automorphisms a of $W$ such that $a(s)$ is a conjugate of $s$ for all $s \in S$ is of finite index in $\operatorname{Aut}(W)$. In particular, there exists an integer $n(W, S)$ such that for any $a \in \operatorname{Aut}(W), a^{n}(s)$ is conjugate to $s$ for all $s \in S$.

Next we prove:

Theorem 28. Suppose that $(W, S)$ is a finitely generated Coxeter system with maximal simplices $\sigma_{1}, \ldots, \sigma_{m}$. If $C$ is the subgroup of all $c \in \operatorname{Aut}(W)$ such that for $i \in$ $\{1, \ldots, m\}$ there exists a $w_{i, c} \in W$ with $c(x)=w_{i, c} x w_{i, c}^{-1}$ when $x \in\left\langle\sigma_{i}\right\rangle$ (i.e., $c$ restricted to $\left\langle\sigma_{i}\right\rangle$ is conjugation by a $w_{i, c} \in W$ depending only on $c$ and $\left.\sigma_{i}\right)$, then $C$ has finite index in $\operatorname{Aut}(W)$.

Proof. If $a \in \operatorname{Aut}(W)$ and $\sigma_{1}, \ldots, \sigma_{m}$ are the maximal simplicies of $(W, S)$, then $a\left(\left\langle\sigma_{i}\right\rangle\right)=w_{i, a}\left\langle\sigma_{\alpha(i)}\right\rangle w_{i, a}^{-1}$ for some $\alpha(i) \in\{1, \ldots, m\}$, by Theorem 26. Observe that $\sigma_{\alpha(j)} \neq \sigma_{\alpha(k)}$ for $k \neq j$, since otherwise

$$
\begin{aligned}
a^{-1}\left(w_{k, a}^{-1}\right)\left\langle\sigma_{k}\right\rangle a^{-1}\left(w_{k, a}\right) & \equiv a^{-1}\left(\left\langle\sigma_{\alpha(k)}\right\rangle\right) \\
& =a^{-1}\left(\left\langle\sigma_{\alpha(j)}\right\rangle\right) \equiv a^{-1}\left(w_{j, a}^{-1}\right)\left\langle\sigma_{j}\right\rangle a^{-1}\left(w_{j, a}\right)
\end{aligned}
$$


implies that $\sigma_{k}=\sigma_{j}$ by Corollary 13. Hence $\alpha$ is a permutation of $(1, \ldots, m)$. If $b \in \operatorname{Aut}(W)$ and $b\left(\left\langle\sigma_{j}\right\rangle\right)=w_{j, b}\left\langle\sigma_{\beta(j)}\right\rangle w_{j, b}^{-1}$ then

$$
b a\left(\left\langle\sigma_{i}\right\rangle\right)=b\left(w_{i, a}\left\langle\sigma_{\alpha(i)}\right\rangle w_{i, a}^{-1}\right)=b\left(w_{i, a}\right) w_{\alpha(i), b}\left\langle\sigma_{\beta(\alpha(i))}\right\rangle w_{\alpha(i), b}^{-1} b\left(w_{i, a}\right)^{-1} .
$$

Hence the map of $\operatorname{Aut}(W)$ into the group of permutations of $(1, \ldots, m)$ defined by $a \mapsto \alpha$ is a homomorphism. If $K$ is the kernel of this homomorphism, then $K$ has finite index in $\operatorname{Aut}(W)$ and for all $a \in K, a\left(\left\langle\sigma_{i}\right\rangle\right)=w_{i, a}\left\langle\sigma_{i}\right\rangle w_{i, a}^{-1}$ for all $i$.

For each $a \in K$ and for each $i$, we see that $a(x)=w_{i, a} \tau_{i}(x) w_{i, a}^{-1}$ for all $x \in$ $\left\langle\sigma_{i}\right\rangle$, where $\tau_{i} \in \operatorname{Aut}\left(\left\langle\sigma_{i}\right\rangle\right)$. The map $q_{i}: K \rightarrow \operatorname{Aut}\left(\left\langle\sigma_{i}\right\rangle\right)$ defined by $a \mapsto \tau_{i}$ is a homomorphism. The main result of [12] shows that $I\left(\left\langle\sigma_{i}\right\rangle\right)$, the inner automorphism group of $\left\langle\sigma_{i}\right\rangle$, has finite index in $\operatorname{Aut}\left(\left\langle\sigma_{i}\right\rangle\right)$. Hence $C \equiv \bigcap_{i=1}^{m} q_{i}^{-1}\left(I\left(\left\langle\sigma_{i}\right\rangle\right)\right)$ is a subgroup of finite index in $\operatorname{Aut}(W)$.

Example 5. The group

$$
\left\langle a, b, c, d: a^{2}=\cdots=d^{2}=1,(a b)^{2},(a c)^{2},(c d)^{2},(b c)^{3},(a d)^{3}\right\rangle .
$$

has a subgroup generated by an edge in the Coxeter diagram that is conjugate to that of a disjoint edge.

The element $b c$ conjugates $\langle a, b\rangle$ to $\langle a, c\rangle$ and the element $a d$ conjugates $\langle a, c\rangle$ to $\langle c, d\rangle$. Hence the subgroup $\langle a, b\rangle$ is conjugate to $\langle c, d\rangle$. Neither group is a maximal FA subgroup.

\section{Visually stable subgroups}

If $(W, S)$ is a finitely generated Coxeter system and $A$ is a special subgroup for this system, then $A$ is $W$-visually stable (or $W-V S$ ) if for any other Coxeter system $\left(W, S^{\prime}\right)$ for $W, A$ is conjugate to a special subgroup for $\left(W, S^{\prime}\right)$.

Knowledge of the visually stable subgroups of a Coxeter group is of interest in several important questions related to the isomorphism problem for Coxeter groups. In particular, this knowledge is useful for "rigidity" questions (see for example [7] and [4] and the references therein) and questions about when reflections are preserved when passing between different Coxeter systems for $W$ (see [1]).

Maximal finite special subgroups are visually stable (see [11]). Clearly $W$ and the trivial group are $W-V S$. The following elementary observation is useful.

Lemma 29. If $H$ is $W-V S$ and $K$ is $H-V S$, then $K$ is $W-V S$.

The next result is a direct corollary to Theorem 26 .

Corollary 30. The maximal FA subgroups of a finitely generated Coxeter group $W$ are $W-V S$. 
Suppose that $(W, S)$ is finitely generated. By Dunwoody's accessibility theorem, $W$ decomposes as a graph of groups $\Lambda$, with finite edge groups such that each vertex group is finite or 1-ended. Suppose that $\Psi$ is a reduced visual decomposition for $(W, S)$ derived from $\Lambda$ as given in Theorem 1 . Any finite or 1-ended subgroup of $W$ is a subgroup of a conjugate of a vertex group of $\Psi$ and so by Theorem 19 there is a bijection $\gamma$ from the vertices of $\Lambda$ to the vertices of $\Psi$ such that $\Lambda(V)$ is conjugate to $\Psi(\gamma(V))$. Note that the vertex groups of $\Psi$ are finite or maximal 1-ended special subgroups of $(W, S)$. In particular we have

Proposition 31. The maximal 1-ended special subgroups of $(W, S)$ are $W-V S$.

The results of [14] and [15] imply that vertex groups of the JSJ decompositions and strong accessibility splittings considered there are $V S$. One example of these implications is the following

Proposition 32. If $\Psi$ is a visual and irreducible with respect to 2-ended splittings decomposition for a 1-ended Coxeter system $(W, S)$ and $V$ is a vertex of $\Psi$, then $\Psi(V)$ is $W-V S$.

An interesting situation occurs during repeated applications of Lemma 29, Proposition 31 and Proposition 32 to an arbitrary finitely generated Coxeter system. Suppose that $H_{1}$ is a maximal 1-ended special subgroup for $(W, S)$, and $H_{2}$ is a maximal 1ended special subgroup of a vertex group of $\Psi$, a visual irreducible with respect to 2-ended splittings decomposition of $H_{1}$. Then $H_{2}$ may split over a 2-ended group (just not in a way compatible with $\Psi$ ). The vertex groups of an irreducible with respect to 2-ended splittings decomposition of $\mathrm{H}_{2}$ are $\mathrm{W}-V S$. Continuing on this line we have

Theorem 33. Suppose that $(W, S)$ is a finitely generated Coxeter system, and $A \subset S$ is maximal in the set of all $A^{\prime} \subset S$ such that the induced diagram for $A^{\prime}$ is not separated by a subdiagram for a finite or 2-ended special subgroup for $(W, S)$. Then $\langle A\rangle$ is $W-V S$.

\section{A final application}

Theorem 1 and Corollary 16 can be applied to "visually" characterize virtually free Coxeter groups.

Theorem 34. The following conditions are equivalent for any Coxeter system $(W, S)$ with presentation diagram $\Gamma(W, S)$ :

(1) $W$ is virtually free; 
(2) W has a visual graph of groups decomposition in which each vertex group is finite;

(3) a) every complete subgraph of $\Gamma(W, S)$ is that of a finite Coxeter group, and

b) no induced subgraph of $\Gamma(W, S)$ is a circuit of more than three vertices.

Proof. Virtually free groups cannot contain 1-ended subgroups. So by Dunwoody's accessibility theorem, every finitely generated virtually free group is the fundamental group of a graph of groups with finite vertex groups. By Theorem 1, (1) implies (2). Conversely, any graph of groups with finite vertex groups has fundamental group which is virtually free, and (2) implies (1).

Again, as $W$ contains no 1-ended subgroup, Corollary 16 can be applied to show (2) implies (3a). A circuit of more than three vertices determines a 1-ended group.

Now suppose condition (3) holds in $\Gamma(W, S)$. If $\Gamma(W, S)$ were a complete graph then by a) it would be finite. Instead take $x$ and $y$ in $\Gamma(W, S)$ not connected by an edge. Consider a component $K$ of the complement of $s t(x)$ in $\Gamma(W, S)$. Let $S_{1}$ be the vertices in $\operatorname{st}(x)$ adjacent to a vertex in $K$. We claim that any two vertices $a$ and $b$ in $S_{1}$ are connected by an edge in $\Gamma(W, S)$, otherwise a minimal length path from $a$ to $b$ in $S_{1} \cup K$ meeting $S_{1}$ only at its endpoints, together with the edges from $b$ to $x$ and $x$ to $a$ would be a circuit in $\Gamma(W, S)$ of more than three vertices contradicting $\mathrm{b})$. Thus $S_{1}$ is a complete graph, and so generates a finite subgroup $C$. Since $S_{1}$ separates $\Gamma(W, S), W$ splits as a nontrivial visual amalgamated product $A *_{C} B$. Inductively, we obtain that (3) implies (2).

\section{References}

[1] P. Bahls and M. Mihalik, Reflection independence in even Coxeter groups. Geom. Dedicata 110 (2005), 63-80. Zbl 1072.20043 MR 2136020

[2] M. Bestvina and M. Feighn, Stable actions of groups on real trees. Invent. Math 121 (1995), 287-321. Zbl 0837.20047 MR 1346208

[3] N. Bourbaki, Groupes et algèbres de Lie. Chap. IV-VI, Hermann, Paris, 1968. Zbl 0186.33001 MR 0240238

[4] N. Brady, J. P. McCammond, B. Mühlherr, and W. D. Neumann, Rigidity of Coxeter groups and Artin groups. Geom. Dedicata 94 (2002), 91-109. Zbl 1031.20035 MR 1950875

[5] K. S. Brown, Buildings. Springer-Verlag, New York 1989. Zbl 0715.20017 MR 0969123

[6] R. W. Carter, Finite groups of Lie type. John Wiley \& Sons Inc., New York 1985. Zbl 0567.20023 MR 0794307

[7] R. Charney and M. Davis, When is a Coxeter system determined by its Coxeter group? J. London Math. Soc. (2) 61 (2000), 441-461. Zbl 0983.20034 MR 1760693 
[8] M.W. Davis, The cohomology of a Coxeter group with group ring coefficients. Duke Math. J. 91 (1998), 297-314; correction ibid. 95 (1998), 635. Zbl 0995.20022 MR 1600586 Zbl 0995.20023 MR 1658756

[9] W. Dicks and M. J. Dunwoody, Groups acting on graphs. Cambridge Stud. Adv. Math. 17, Cambridge University Press, Cambridge 1989. Zbl 0665.20001 MR 1001965

[10] M. J. Dunwoody, The accessibility of finitely presented groups. Invent. Math. 81 (1985), 449-457. Zbl 0572.20025 MR 807066

[11] W. N. Franzsen, Automorphisms of Coxeter groups. PhD thesis, University of Sydney, 2001.

[12] R. B. Howlett, P. J. Rowley, and D. E. Taylor, On outer automorphism groups of Coxeter groups. Manuscripta Math. 93 (1997), 499-513. Zbl 0888.20023 MR 1465894

[13] R. C. Lyndon and P. E. Schupp, Combinatorial group theory, Ergeb. Math. Grenzgeb. 89, Springer-Verlag, Berlin 1977. Zbl 0368.20023 MR 0577064

[14] M. L. Mihalik and S. Tschantz, Strong accessibility of Coxeter groups over minimal splittings. Preprint 2005.

[15] M. L. Mihalik and S. Tschantz, JSJ-decompositions of Coxeter groups over virtually abelian splittings. Preprint 2005.

[16] P. Scott and T. Wall, Topological methods in group theory. In Homological group theory (Durham, 1977), London Math. Soc. Lecture Note Ser. 36, Cambridge University Press, Cambridge 1979, 137-203. Zbl 0423.20023 MR 0564422

[17] J.-P. Serre, Trees. Springer-Verlag, Berlin 1980. Zbl 0548.20018 MR 0607504

[18] J. Stallings, Group theory and three-dimensional manifolds. Yale University Press, New Haven 1971. Zbl 0241.57001 MR 0415622

Received April 22, 2008; revised June 9, 2008

M. Mihalik, Department of Mathematics, Vanderbilt University, Nashville, TN 37240,

U.S.A.

E-mail: michael.1.mihalik@ vanderbilt.edu

S. Tschantz, Department of Mathematics, Vanderbilt University, Nashville, TN 37240,

U.S.A.

E-mail: tschantz@math.vanderbilt.edu 